\title{
Desarrollo del curso genética general mediado por TIC durante el primer cuatrimestre del 2020
}

\section{Development of the general genetics course mediated by TIC during the first quarter of 2020}

\author{
Virginia Aliverti \\ https://orcid.org/0000-0002-7171-0734 \\ virginia.aliverti@fcv.unlp.edu.ar \\ Facultad de Ciencias Veterinarias | \\ Universidad Nacional de La Plata | Argentina
}

\section{Julio De Luca}

https://orcid.org/0000-0003-2711-1192

delucajc@hotmail.com

Facultad de Ciencias Veterinarias |

Universidad Nacional de La Plata | Argentina

\author{
Sebastian Picco \\ https://orcid.org/0000-0002-2904-2365 \\ sjpicco@gmail.com \\ Facultad de Ciencias Veterinarias | \\ Universidad Nacional de La Plata | Argentina
}

\author{
Pilar Peral García \\ https://orcid.org/0000-0003-3015-4628 \\ peralgarciapilar@gmail.com \\ Facultad de Ciencias Veterinarias | \\ Universidad Nacional de La Plata | Argentina
}

\section{RESUMEN}

Debido al Aislamiento Social, Obligatorio y Preventivo (ASPO) por el COVID- 19, la Universidad Nacional de La Plata comunicó a sus docentes la necesidad de mantener la continuidad pedagógica en todas sus dependencias. En la Facultad de Ciencias Veterinarias las TIC se comenzaron a utilizar ampliamente en los últimos años para mejorar los modelos formativos y organizativos que sustentan el aprendizaje. El objetivo fue describir la experiencia en el desarrollo del curso Genética General durante el ASPO- COVID-19, mediado por TIC y analizar el desempeño de las cohortes 2019 (cursada presencial) y 2020 (cursada virtual). Se utilizó el entorno virtual Moodle 3.1 como soporte didáctico. Las Actividades Presenciales Obligatorias se convirtieron en Actividades Virtuales Obligatorias. El total de alumnos en estudio fue de 296 de los cuales 150 corresponden a la cursada del 2019 y 146 a la del primer cuatrimestre 2020. Cuando se compararon las condiciones de cada alumno, no se encontraron diferencias significativas entre cantidad de promocionados, aprobados, desaprobados y ausentes entre los dos grupos. El uso de las TIC durante el ASPO nos permitió desarrollar el curso sin mayores inconvenientes; fortaleciendo la relación entre docentes y estudiantes.

PALABRAS CLAVE

TIC,

COVID-19,

Genética General. 
KEY WORDS

TIC,

COVID-19,

General Genetics.

\section{ABSTRACT}

Due to the Social, Mandatory and Preventive Isolation (ASPO) by COVID-19, the National University of La Plata communicated to its teachers the need to maintain pedagogical continuity in all its dependencies. In the Faculty of Veterinary Sciences, TIC began to be widely used in recent years to improve the training and organizational models that support learning. The objective was to describe the experience in the development of the General Genetics course during the ASPO-COVID-19, mediated by TIC and to analyze the performance of the cohorts 2019 (attended in person) and 2020 (attended virtual). The virtual environment Moodle 3.1 was used as didactic support. Mandatory Face-to-Face Activities became Mandatory Virtual Activities. The total number of students under study was 296 of which 150 correspond to the one studied in 2019 and 146 to the first semester 2020. When the conditions of each student were compared, no significant differences were found between the number of promoted, approved, disapproved and absent between the two groups. The use of TIC during the ASPO allowed us to develop the course without major inconveniences; strengthening the relationship between teachers and students. 


\section{INTRODUCCIÓN}

Los enfoques actuales del desarrollo consideran a la educación como una inversión en capital humano y le atribuyen un papel fundamental en el logro de una mayor equidad social. En ese modelo de sociedad, la universidad posee una responsabilidad social innegable, ya que resulta un faro permanente, tomado como referente por los distintos actores sociales. Participa en la tarea de construir un nuevo tipo de vida y de hombre y es imprescindible crear en ella nuevos modos y estructuras educativas con una determinada programación educativa y una coherente metodología del aprendizaje y enseñanza (Nassif, 1984: Cap. 14). Buscar los conocimientos dinámicos y fértiles, aquellos que abren el acceso a nuevos conocimientos y entrenan la mente para el aprendizaje, es una de las grandes tareas pedagógicas en un mundo sacudido por cambios vertiginosos y reclamos de toda índole. La digitalización y la automatización han provocado una profunda revolución, caracterizada especialmente por la aparición de dispositivos multimedia y por una expansión espectacular de las redes telemáticas. Los sistemas expertos y la inteligencia artificial aumentan vertiginosamente la interactividad. La velocidad de procesamiento de la información crece constantemente, así como la capacidad casi ilimitada de almacenamiento. En cualquier caso, no es posible entender la configuración de esta sociedad sin la influencia de la información (Blázquez Entonado, 2001).

Los nuevos planteamientos curriculares en la universidad demandan una integración de los contenidos y de los saberes, una actitud renovada de los docentes frente a las estrategias metodológicas y 
una relación más propia del saber con sus objetos de conocimiento. Enseñar no es transferir conocimiento sino crear las posibilidades para su propia producción o construcción (Villafuerte, Cevallos, y Vidal, 2020: 136). La clave del proceso enseñanza- aprendizaje reside en las relaciones que se establecen entre los tres elementos que conforman un triángulo interactivo, o triangulo didáctico: el contenido, que es objeto de enseñanza y aprendizaje, la actividad educativa e instruccional del docente y las actividades de aprendizaje de los estudiantes.

\section{Los nuevos planteamientos curriculares en la universidad de- mandan una integración de los contenidos y de los saberes, una actitud renovada de los docentes frente a las estrategias metodológicas y una relación más propia del saber con sus objetos de conocimiento.}

Las nuevas tecnologías y los espacios virtuales, se constituyen en una forma de pensar la educación de manera diferente, no tradicional. Entre todas las tecnologías creadas por los seres humanos, las relacionadas con la capacidad de transmitir información tienen especial importancia, dado que afectan los ámbitos de la actividad de las personas. Esta nueva forma implica por un lado una incorporación tecnológica, y por otro una reflexión pedagógica para el desarrollo de la relación docente- alumno- contenidos (Sigalés, 2001).

En una Universidad presencial, la tecnología suele usarse como complemento y su forma de aplicación es más fácilmente adaptable ya que las experiencias de uso de las TIC (Tecnologías de la Información y Comunicación) no necesariamente comprometen a toda la organización. El aula virtual permite tener el registro de todo el proceso de cada estudiante mediante diferentes intervenciones y producciones. Precisamente por ello, es importante plantear y sistematizar la innovación para mejorar los modelos formativos y organizativos que sustentan el aprendizaje. Además, el aprendizaje con soporte tecnológico se está desarrollando con mucha rapidez en el ámbito de la educación superior, por lo que incorporar la innovación constituye más que un indicador de calidad, una necesidad ineludible (Gros Salvat y Fernández, 2010). Ante esta aparente factibilidad que el sector educativo parece connotar ante la virtualización de la tarea, es posible afirmar que resulta indiscutible que las herramientas sincrónicas (chats, videoconferencias en tiempo real) otorgan mayor posibilidad de interacción social y cognitiva atendiendo especialmente al componente tecnológico, la conectividad, la aplicación.

En Argentina, a partir de la aplicación del Aislamiento Social Preventivo y Obligatorio (ASPO) decretado por el gobierno nacional el 
19 de marzo de 2020, con el objetivo de disminuir la tasa de contagio de COVID-19, el mundo del trabajo se vio atravesado por un sinfín de modificaciones. En el ámbito universitario y considerando la autarquía propia de las casas de altos estudios, hubo diferentes posiciones. A la par que la Universidad de Buenos Aires reprogramaba su calendario académico, posponiendo el inicio de clases para el mes de junio (Cafferata, 2020), otras universidades se deciden por la continuidad (Fernández, 2020) y entre ellas, la Universidad Nacional de La Plata ratifica su calendario y comunica a sus docentes sobre la necesidad de mantener la continuidad pedagógica en todas sus Facultades (UNLP); e impulsó la necesidad de readecuar y adoptar TIC para el correcto desarrollo de las actividades académicas.

En la Facultad de Ciencias Veterinarias de la Universidad Nacional de La Plata (FCV-UNLP), las TIC, se comenzaron a utilizar ampliamente en los últimos años para mejorar los modelos formativos y organizativos que sustentan el aprendizaje. Se utiliza como soporte de enseñanza el entorno virtual Moodle $e^{\text {ii". }}$ Las TIC y sus potencialidades nos retan a la creación de espacios educativos, sean presenciales o virtuales, que promuevan la interacción personal, el debate plural, la reciprocidad inmediata y completa de los intercambios, la pluralidad de los puntos de vista, las relaciones directas entre docentes y alumnos. Lo más frecuente es proponer los medios tecnológicos como posibles instrumentos didácticos poniendo el énfasis en las habilidades necesarias para su eficiente utilización. Pero no nos preguntamos sobre los modelos pedagógicos idóneos o convenientes para el uso de estas tecnologías, ni los modos más adecuados para su introducción en los currículos (Blázquez Entonado, 2001).

\section{Las TIC y sus potencialidades nos retan a la creación de espa- cios educativos, sean presenciales o virtuales, que promuevan la interacción personal, el debate plural, la reciprocidad inme- diata y completa de los intercambios, la pluralidad de los pun- tos de vista, las relaciones directas entre docentes y alumnos.}

\footnotetext{
i Se efectuó atento a la excepcionalidad de la situación, aún sin contar con una normativa específica para el desarrollo curricular en modalidad e-learning, ya que estatutariamente, las carreras de grado de la FCV-UNLP son presenciales.

ii Moodle es un software diseñado para ayudar a los educadores a crear cursos en línea de alta calidad y entornos de aprendizaje virtuales. Tales sistemas de aprendizaje en línea son algunas veces Ilamados VLEs (Virtual Learning Environments) o entornos virtuales de aprendizaje. La palabra Moodle originalmente es un acrónimo de Modular Object-Oriented Dynamic Learning Environment (Entorno de Aprendizaje Dinámico Orientado a Objetos y Modular). Una de las principales características de Moodle sobre otros sistemas es que está hecho en base a la pedagogía social constructivista, donde la comunicación tiene un espacio relevante en el camino de la construcción del conocimiento; siendo el objetivo generar una experiencia de aprendizaje enriquecedora. Las principales ventajas de Moodle para educadores y capacitadores: sistema escalable en cuanto a cantidad de alumnos, posilibidad de diversos métodos de evaluación y calificación, accesibilidad y compatibilidad desde cualquier navegador web, independiente del sistema operativo (http://www.entornos.com.ar/moodle).
} 


\section{DESARROLLO DEL CURSO GENETICA GENERAL}

El Plan de Estudios 406/14 de la Carrera de Ciencias Veterinarias de la Universidad Nacional de La Plata (FCV-UNLP) plantea un ciclo generalista integrado por 54 cursos obligatorios (3.600 horas) y un ciclo orientado (orientación profesional) integrado por tres cursos optativos de 40 horas cada uno y las practicas pre-profesionales de 300 horas (420 horas totales). El perfil del profesional egresado, es el que, en función del mejoramiento de la calidad de vida y el desarrollo humano sustentable, imbuido en un espíritu ético, científico y humanístico; sea capaz de ejecutar acciones tendientes a la previsión, planificación y resolución de la problemática planteada en diferentes ámbitos de la realidad socio-cultural y del medio ambiente, con relación a todo lo que directa o indirectamente sea atinente a las especies animales.

El curso de Genética General se concibe como la introducción de los alumnos de veterinaria al estudio y comprensión de los modelos de herencia y de la expresión génica, que constituyen la base teórica necesaria para acceder a los contenidos de genética cuantitativa y de genética de poblaciones. Tiene una carga horaria total de 40 horas, divididas en doce Actividades Presenciales Obligatorias (APO), una por semana. Cada encuentro es de modalidad presencial (teórico práctico). El objetivo fue describir la experiencia en el desarrollo del curso Genética General durante el ASPO-COVID-19, mediado por TIC y analizar el desempeño de las Cohortes 2019 (cursada presencial) y 2020 (cursada virtual).

\section{La planificación curricular, bibliografía, links de las presen- taciones teóricas y la actividad práctica se subió al entorno virtual Moodle 3.1; el cual se utilizó como soporte didáctico.}

Por medio de resoluciones de presidencia de la UNLP, la no realización de instancias presenciales por el ASPO producto de la pandemia del COVID-19, impuso la necesidad de readecuar y adoptar TIC para el correcto desarrollo de las actividades académicas. En consecuencia, a mediados de marzo, se modificó la metodología para el desarrollo del curso durante el primer cuatrimestre del 2020. La planificación curricular, bibliografía, links de las presentaciones teóricas y la actividad práctica se subió al entorno virtual Moodle 3.1; el cual se utilizó como soporte didáctico. Las APO, se convirtieron en Actividades Virtuales Obligatorias (AVO), y consistieron en presentaciones teóricas a cargo de los profesores, las cuales fueron grabadas y subidos a YouTube, y por Zoom las actividades prácticas a cargo de docentes, los miércoles de 9 a 12 hs. Una vez desarrollados todos los contenidos del curso distribuidos en $12 \mathrm{AVO}$, se tomó una evaluación integradora, la que tuvo tres instancias para su aprobación, de acuerdo a la planificación curricular. Esta evaluación es un instrumento objetivo de medición numérica, cuyo propósito es que el alumno demuestre el 
desarrollo progresivo de su capacidad de interpretación e integración de los conceptos vistos durante la cursada y aplicados a la resolución de problemáticas del campo veterinario. La aprobación de la cursada ordinaria requiere la obtención de una nota de cuatro(4) puntos o más en la evaluación integradora, la obtención de siete (7) o más puntos le permite al alumno promocionar el curso, mientras que el puntaje por debajo de cuatro puntos es considerado insuficiente para aprobar. Los resultados fueron analizados mediante estadística descriptiva y comparados utilizando el análisis de Ji Cuadrado con el programa Statgraphics Centurión XVI.

\section{RESULTADOS}

Se compararon las notas finales subidas al SIU Guaraní de las Comisiones A y B del Curso de Genética General de la Carrera de Ciencias Veterinarias de la UNLP. El total de alumnos en estudio fue de 296 de los cuales 150 corresponden a la cursada del 2019 y 146 a la del primer cuatrimestre 2020. Cuando se compararon las condiciones de cada alumno, no se encontraron diferencias significativas entre cantidad de Promocionados, Aprobados, desaprobados y Ausentes entre los dos grupos (Tabla 1 y Figura 1).

\begin{tabular}{|l|l|l|l|}
\hline Prueba & Estadístico & Gl & Valor-P \\
\hline Chi-Cuadrada & 5,886 & 3 & 0,1173 \\
\hline
\end{tabular}

Tabla 1: Resultado del análisis estadístico Chi -Cuadrada, comparación de cohortes 2019 y 2020.

Diagrama de Barras para Cohorte según Resultado

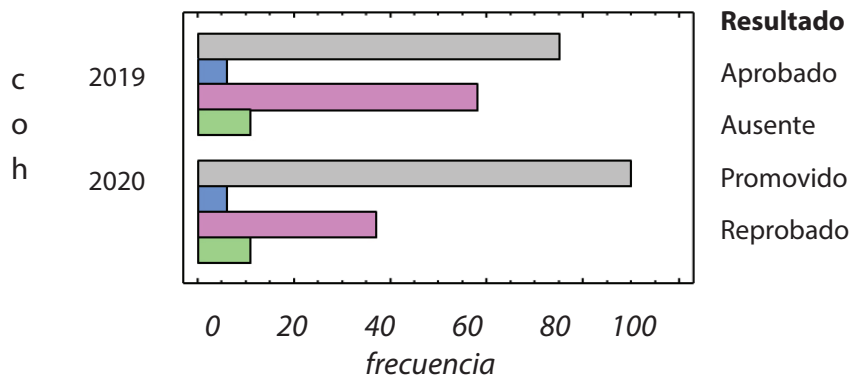

Figura 1: Resultados cohortes 2019 y 2020.

\section{CONCLUSIONES}

A través del presente trabajo, hemos realizado una descripción de la experiencia en el uso de TIC para el desarrollo del curso de Genética General durante el primer cuatrimestre del 2020. Además, analizamos 
el desempeño de los alumnos de la cohorte 2019 (cursada presencial) y 2020 (cursada a distancia).

El uso de las TIC durante el ASPO-COVID-19 nos permitieron desarrollar el curso sin mayores inconvenientes; fortaleciendo la relación entre docentes y estudiantes.

Por su parte, la implementación de una evaluación mediada por TIC para que los alumnos acrediten el curso, fue satisfactoria. Al comparar los resultados de las cohortes 2019 y 2020 nos permitió identificar virtudes y defectos en la implementación de TIC y de esa manera articular nuevas innovaciones.

Si bien el curso de Genética General es cuatrimestral; actualmente nos encontramos desarrollando el mismo por solicitud de las autoridades de la FCV-UNLP para aquellos alumnos que consiguieron la regularidad en el primer cuatrimestre.

Los avances tecnológicos producidos en las últimas décadas han determinado que sea necesario hacer uso de los recursos informáticos en muchos aspectos de la vida diaria. En una Universidad presencial, el uso de tecnologías digitales como herramienta pedagógica representa una propuesta innovadora. En la FCV-UNLP, se utiliza como soporte de enseñanza el entorno virtual Moodle desde hace varios años, ampliando el concepto de clase/aula, permitiendo complementar la clase presencial con el uso de actividades virtuales.

Más allá de las inéditas posibilidades que las TIC ofrecen para representar, procesar, transmitir y compartir información, su potencialidad educativa está

estrechamente ligada con las características y propiedades del entorno simbólico que estas tecnologías ponen a disposición del docente y del estudiante. 


\section{BIBLIOGRAFÍA}

Blázquez Entonado, F. (Coord.) (2001). “Sociedad de la información y comunicación". Junta de Extremadura. Consejería de Educación, Ciencia y Tecnología Dirección General de Ordenación, Renovación y Centros. Mérida.

Cafferata, M. (5 de abril de 2020) “Las clases en la UBA comenzarán el $1^{\circ}$ de junio". Página 12. Recuperado de https://www.pagina12.com.ar/257588- lasclases-en-la-uba-comenzaran-el-1-de-junio.

Fernández, M. (6 de abril de 2020). “Tras la decisión de la UBA, ¿Cómo continuarán las clases en otras universidades?" Infobae. Recuperado de https:// www.infobae.com/educacion/2020/04/06/tras-la-decision-de-la-uba-comocontinuara-las-clases-en-otras-universidades/.

Gros Salvat, B; López Fernández, O; Castillo Navarro, J y González Prieto, F. (2010). “Entornos Virtuales de Comunicación On-line en la Enseñanza Superior". Disponible en: https://recursos.educoas.org/publicaciones/entornos-virtuales-de-comunicaci-n-line-en-la-ense-anza-superior

Nassif, R. (1984). “Teoría de la Educación”. Cap. 14: Dialéctica de la educación. Madrid: Cincel-Kapelusz.

Sigalés, C. (2001). "X Encuentro Internacional de Educación a Distancia. El potencial interactivo de los entornos virtuales de enseñanza y aprendizaje en la educación a distancia". Guadalajara, México.

Universidad Nacional de La Plata (2020). “Comunicado: La UNLP ratifica la vigencia del calendario académico". Recuperado htts://unlp.edu.ar/coronavirus/ comunicado-la-unlp-ratifica-la-vigencia-del- calendario-academico-17703

Villafuerte, J., Cevallos, Y. P., y Vidal, J. O. B. (2020). “Rol de los docentes ante la crisis del COVID-19. Una mirada desde el enfoque humano". REFCalE: Revista Electrónica Formación y Calidad Educativa. ISSN 1390-9010, 8 (1), 134-150.

Moodle http://www.entornos.com.ar/moodle 